\title{
Evaluation and Correction of Cable Phase Stability in High Frequency Near-Field Measurement
}

\author{
Jan VANCL, Petr ČERNÝ, Zbyněk ŠKVOR, Miloš MAZÁNEK \\ Dept. of Electromagnetic Field, Czech Technical University, Technická 2, 16627 Praha, Czech Republic \\ vanclj1@fel.cvut.cz, xcernyp1@fel.cvut.cz, skvor@fel.cvut.cz, mazanekm@fel.cvut.cz
}

\begin{abstract}
In case of the near-field measurement, the radiated field by the measured antenna is received by twodimensional scanner in defined points. During the process of the field scanning, the cable between the VNA and the scanner antenna is moved and its parameters are changed. This paper deals with the evaluation of the cable parameters changes. The dominant change is represented by the phase deviation of the cable transmission coefficient.
\end{abstract}

The paper also proposes the method of the cable errors correction and evaluation of the correction method efficiency. The correction can be based on the full calibration at the reference plane of the scanner antenna feeding in all points of scanned field. The aforementioned approach is more time consuming than the proposed method, where the full calibration is performed in one selected point, while the correction of the cable parameters is based on the estimation based on the input impedance of the cable loaded by the scanner antenna.

\section{Keywords}

Near-filed, radiation pattern, cable phase stability, VNA, 2D plane scanner, error two-port.

\section{Introduction}

Commonly used high directive antennas or phased arrays have very huge far-field region. The measurement of the antenna patterns is more complicated or very expensive. The antenna pattern measurement can be performed using the near-field method. The electromagnetic filed radiated by the measured antenna is scanned in the near-field region and the captured field distribution is transformed into the far-field antenna patterns [1]. It is necessary to capture all complex parameters, thus the vector network analyzer (VNA) is used for the characteristic measurement.

The radiated field is commonly captured in the twodimensional plane $(x, z)$, cylinder $(\phi, z)$ or sphere $(\phi, \vartheta)$.
During the scanner antenna movement, the cable between the scanner antenna and the VNA is moved. Thus, the parameters of this cable are also changed. This change can be so high that the distortion of the transformed far-filed antenna pattern can exceed the feasible level.

The cable error can be corrected by the full calibration using open, short and match calibers (OSM) in all measured points during the scanning. This procedure is very time consuming, because it is necessary to perform four scanner measurements (three calibers and the scanner antenna) in all points. It is also possible to use microwave switches and E-Cal devices in order to minimize the number of performed scanner measurement to one. However, the needed equipment does not represent commonly used devices and, at the same time, do not exist for the higher frequencies. The cable errors can be also minimized by using of the cables with the high degree of the phase stability. The costs of these cables are very high, thus the latter stay out of reach of the majority of the research groups.

The proposed correction method, stated below, was elaborated in order to minimize the cable phase error with minimal number of measurement. The proposed method can be easily extended and applied also in case of the nearfiled measurement of antennas patterns (which is not the field of interest of this paper).

\section{Correction Method}

The cable can be characterized by the error two-port [2] in each point of measurement array of the scanned area. The error two-port of the cable is obtained by OSM calibration of VNA on the end of cable in case of the ideal VNA (calibrated VNA); see Fig. 1.

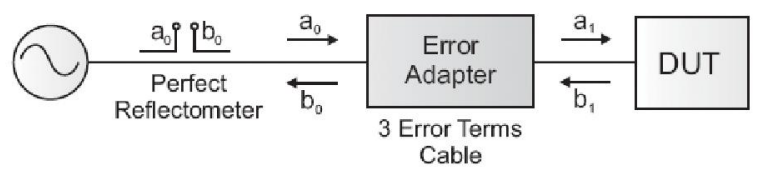

Fig. 1. Basic measurement scheme. 
Under thos condition, the error two-ports (ETP) of cable, which transforms measured $\mathrm{S}_{11}$ parameter into the calibrated VNA (Fig.1), can be calculated by means of the following equation [3]:

$$
\left[\begin{array}{c}
e_{00} \\
e_{11} \\
\Delta_{e}
\end{array}\right]=\left[\begin{array}{ccc}
1 & \Gamma_{M 1} \Gamma_{A 1} & -\Gamma_{A 1} \\
1 & \Gamma_{M 2} \Gamma_{A 2} & -\Gamma_{A 2} \\
1 & \Gamma_{M 3} \Gamma_{A 3} & -\Gamma_{A 3}
\end{array}\right]^{-1} *\left[\begin{array}{c}
\Gamma_{M 1} \\
\Gamma_{M 2} \\
\Gamma_{M 3}
\end{array}\right],
$$

which are derived from

$$
\Gamma_{M}=\frac{b_{0}}{a_{0}}=\frac{e_{00}-\Delta_{e} \Gamma_{A}}{1-e_{11} \Gamma_{A}},
$$

where

$$
\Delta_{e}=e_{00} e_{11}-\left(e_{10} e_{01}\right) \text {. }
$$

2.

The parameters of error two-ports are shown in Fig.

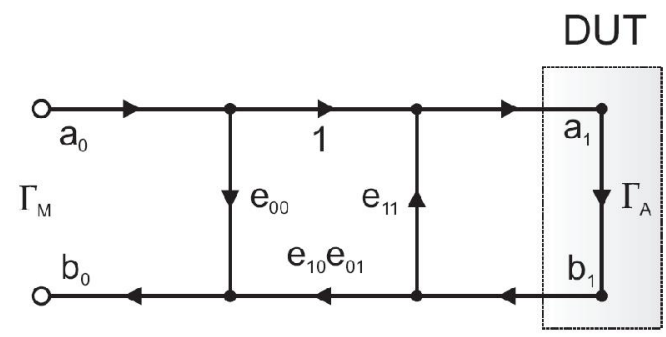

Fig. 2. Parameters of error two-port.

The OSM calibration is made on the edge of the cable in the first point of measurement array. The scanner antenna is measured in each point of measurement array. In the first point (calibration) of measurement array, the actual reflection coefficient of component can be calculated as follows:

$$
\Gamma_{A}(1)=\frac{\Gamma_{M}(1)-e_{00}(1)}{\Gamma_{M}(1) e_{11}(1)-\Delta_{e}(1)},
$$

where the number in the brackets stands for the positions in measurement array.

Provided that, the $\mathrm{e}_{00}$ and $\mathrm{e}_{11}$ parameters of error twoport of cable remain constant for each point of measurement array, parameter $\Delta_{\mathrm{eC}}$ can be calculated according to the following equation

$$
\Delta_{e C}(n)=\frac{\Gamma_{M}(n) \Gamma_{A}(1) e_{11}(1)+e_{11}(1)-\Gamma_{M}(n)}{\Gamma_{A}(1)},
$$

in each point in measurement array. Then the corrected reflection coefficient can be calculated as

$$
\Gamma_{A}(n)=\frac{\Gamma_{M}(n)-e_{00}(1)}{\Gamma_{M}(n) e_{11}(1)-\Delta_{e C}(n)} .
$$

\section{Experimental Measurement}

The vector network analyzer Agilent E8364A and calibers Agilent $85056 \mathrm{~K}$ were used for the experimental measurement. Firstly, the VNA was calibrated by using the OSM method and the parameters of calibers were measured. All measurements were performed within the frequency band ranging from 36 to $38.5 \mathrm{GHz}$. Secondly, the cable (Rosenberger RTK040, $2 \mathrm{~m}$ ) was connected between VNA and measurement array. The input impedance of the cable was measured, whereas the OSM calibers and scanner antenna were sequentially connected at the end of the cable, in every point of the measurement array.

The average standard deviations (SD) of the cable error two-port parameters follow:

$$
\begin{aligned}
& S D\left(e_{00}\right)=0.004 \\
& S D\left(e_{11}\right)=0.004 \\
& S D\left(e_{10} e_{01}\right)=0.05
\end{aligned}
$$

The parameter $S D\left(e_{1} e_{01}\right)$ is twelve times higher than the other parameters. As a result, it is acceptable to neglect the parameters $S D\left(e_{00}\right)$ and $S D\left(e_{11}\right)$ in the correction. The standard deviation of the amplitude of the reflection coefficient for different points was, on average, equal to 0.01 , which corresponds to the accuracy of VNA measurement. As a consequence, the correction of the amplitude turns out to be useless. The standard deviation of the phase of reflection coefficient equaled, on average, $10^{\circ}$.

The standard deviations of the measured reflection coefficients of the components (short, open and waveguide aperture) in every point of the measurement array are depicted in Fig. 3, 4, 6 and 7. All lines in figures show the standard deviations of the measured impedances, solved from all points of the measurement array. The measured impedance (short, open or aperture antenna) is transformed through the solved error two-port from the impedance measured at the beginning of the cable.

The SD, where the ETP is calculated only for one single point of the measurement array and each measurement was transformed through the aforementioned ETP, is indicated by the green line. The SD, where the ETPs are calculated from the OSM calibers measured in all points, is depicted by the blue line. The SD, where the ETP is calculated only for one single point of the measurement array and corrected in all points, is represented by the red line. The correction is based on the impedance (short, open, aperture antenna located at the cable end) changes measured at the cable beginning.

In case of the short as the correction component, the $\mathrm{SD}$ of the open transformed through the ETP solved in all points is approximately fourteen times smaller $\left(0.7^{\circ}\right)$ than the $\mathrm{SD}$ of the open transformed through the ETP solved only in one point. Moreover, it is approximately seven times smaller $\left(1.5^{\circ}\right)$ than the SD of the open transformed 
through the corrected ETP solved only in one point; see Fig. 3 and 4.

The high value of the SD of phase is caused, at some frequencies, by the small value of the reflection coefficient of the waveguide aperture; see Fig.5. In case that the correction component is waveguide aperture (Fig.6,7), the correction method efficiency is unsatisfactory at the frequencies, where the reflection coefficient of waveguide aperture is smaller than 0.15; see Fig.5.
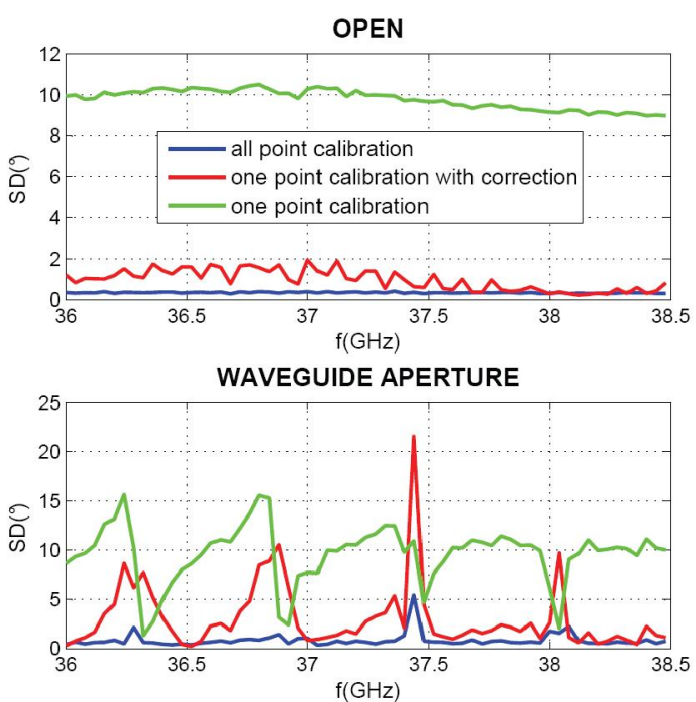

Fig. 3. Standard deviation of reflection coefficient phase of open and waveguide aperture. Measurement of reflection coefficient of short was used for correction.
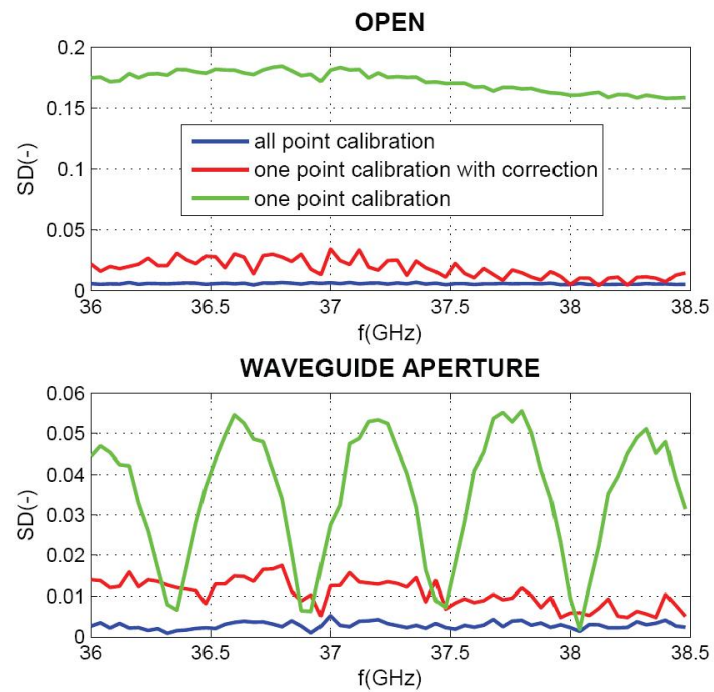

Fig. 4. Standard deviation of reflection coefficient of open and waveguide aperture. Measurement of reflection coefficient of short was used for correction.

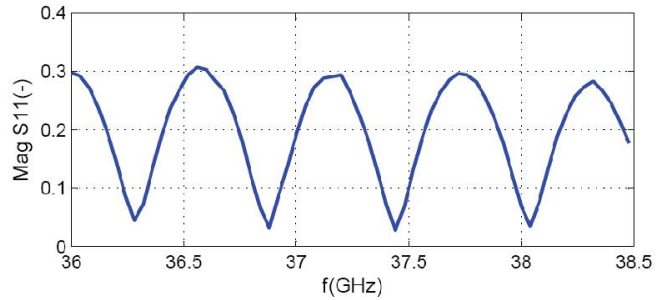

Fig. 5. Magnitude of reflection coefficient of waveguide aperture.
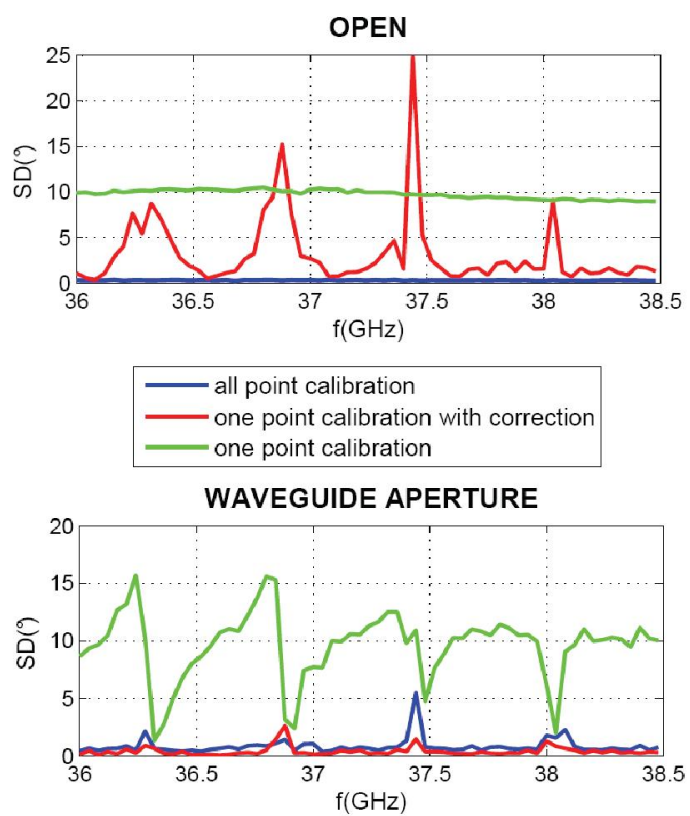

Fig. 6. Standard deviation of reflection coefficient phase of open and waveguide aperture. Measurement of reflection coefficient of waveguide aperture was used for correction.
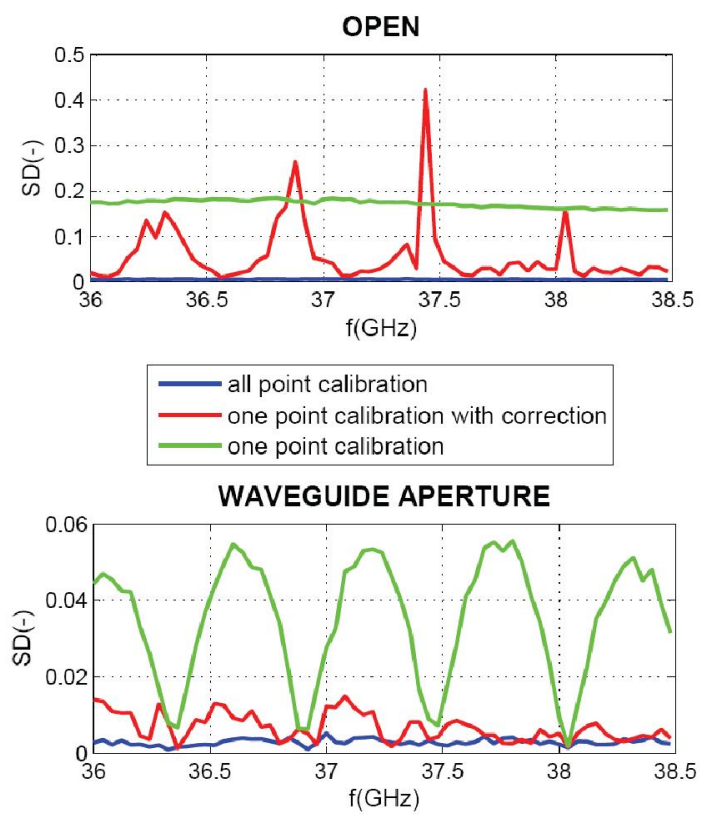

Fig. 7. Standard deviation of reflection coefficient of open and waveguide aperture. Measurement of reflection coefficient of waveguide aperture was used for correction. 


\section{Conclusion}

For the near-field measurement, the cable parameters were measured and the changes were evaluated in the frequency band of up to $40 \mathrm{GHz}$. Changes of the cable reflections and the amplitude of the transmission equal approximately to the reflection measurement error of the VNA. On the other hand, the SD of phase changes of the cable transmission is approximately equal to $10^{\circ}$.

In case of the OSM calibration in all points of the measured array, the SD of phase changes is dramatically lower. It amounts approximately to $0.7^{\circ}$. The abovementioned method is very time consuming.

This paper proposes the simplified correction method and analyzes its advantages as well as disadvantages. The OSM calibration was performed only in one single position. The cable phase error is corrected using the measured deviance of the cable/antenna impedance. The $\mathrm{SD}$ of phase changes equals approximately $1.5^{\circ}$. Although the latter is approximately two times higher, the time of the measurement is much shorter. This correction is successful only in case the scanner antenna reflections are better than 0.15 .

\section{Acknowledgements}

The research has been conducted at the Department of Electromagnetic Field of Czech Technical University in Prague and supported by Research Programme MSMT6840770015 "Research of Methods and Systems for Measurement of Physical Quantities and Measured Data Processing". The measurement was supported by the project Research in the Area of the Prospective Information and Navigation Technologies MSM 6840770014 .

\section{References}

[1] BALANIS, C. A. Antenna Theory, analysis and Design. John Wiley and Sons, 1997

[2] RYTTING, D. An Analysis of Vector Measurement Accuracy Enhancement Techniques," In Proceedings of Hewlett-Packard RF \& Microwave Symposium. March 1982, pp.16-20.

[3] SATLER, M. J., RIDLER, N. M., HARRIS, P. M. Over-determined calibration schemes for RF network analysers employing generalized distance regression. In Proceedings of the 62th ARFTG Conferenc., Boulder, 2003, pp. 127-140. 